\title{
El estudio de cambio de código en un corpus de breves textos literarios
}

\author{
An Vande Casteele, Lauren Dethier \\ Vrije Universiteit Brussel
}

\begin{abstract}
The present study investigates the different types of code-switching in a literary corpus in order to evaluate the degree of code change. A corpus-based approach is adopted to identify the intersentential and intrasentential changes and the tag-switching cases in a literary corpus of short stories written in Spanglish. In the present article a case study on the use of code-switching in Más estrellas que en el cielo, a short story written by Alberto Fuguet, is presented in order to illustrate how we proceed. The final aim of the study is to position each example, and then in a more general way, each text, on a scale in which the two codes, Spanish and English, are located in the extreme points and, as such, to determine the degree of code switching.
\end{abstract}

Keywords: Spanglish, code-switching, corpus analysis, Matrix Language Frame

\section{Introducción}

Hoy en día, el multilingüismo es omnipresente y hay un creciente interés en todo fenómeno relacionado con lenguas en contacto. Así, el presente trabajo se enmarca en este mismo tema del contacto de lenguas y propone investigar las distintas manifestaciones del cambio de código inglés-español. El estudio ofrece un análisis basado en un corpus de breves textos literarios y persigue proporcionar pistas para una clasificación, una categorización de distintos tipos de textos en spanglish y del grado de cambio de código que presentan. Cabe subrayar que el Spanglish no solo se delimita al dominio lingüístico propiamente dicho, como modalidad de habla que da cuenta de una convergencia del inglés y del español, sino que, como observa Silvia Betti (2009), es el resultado de un encuentro de dos mundos, dos culturas y dos idiomas: el hispánico y el anglosajón.

De manera general, el cambio de código puede definirse como una alternancia de idiomas en un mismo texto o discurso. De acuerdo con Lesley Milroy y Pieter 
Muysken (1995) suelen distinguirse tres tipos de alternancias: el cambio de código intersentencial, intrasentencial y el tag-switching. Como marco del estudio conviene señalar también el modelo elaborado por Carol Myers-Scotton (2001): el modelo del Matrix Language Frame que diferencia entre la lengua matriz y la lengua insertada. En el caso particular del spanglish se trata de una mezcla de los códigos español e inglés.

Ahora bien, como indicado, en este artículo se presenta una aproximación basada en un corpus literario de breves cuentos escritos en spanglish y la finalidad del estudio consiste en llegar a una clasificación de los diferentes usos del spanglish. Para el análisis se procede de la manera siguiente: de cada texto individual en el corpus se ofrece un estudio detenido de los patrones típicos en el cambio de código y en una etapa posterior se procede a un análisis global del corpus en la totalidad. El propósito del presente artículo consiste en ofrecer un estudio de caso en que proporcionamos un examen detenido de los diferentes tipos de cambio de código presentes en la obra Más estrellas que en el cielo escrita por Alberto Fuguet. Se comentará el grado de variedad y los distintos tipos de alternancia que pueden observarse en la obra. En fin, se trata, pues, de un ejemplo concreto que ilustra cómo se procede en el análisis.

\section{Definición de cambio de código}

El spanglish es, como lo indica Silvia Betti (2009: 104), fruto de un encuentro de dos mundos, dos culturas, dos idiomas: "El spanglish, de span- (spanish) más glish (english), es el fruto, pues, del encuentro (o del choque) entre dos mundos, dos sensibilidades, dos culturas e idiomas: el hispánico y el anglosajón". Además, como lo apunta Ilan Stavans (2000a: 557), responde a una necesidad comunicativa, sirve como un recurso expresivo que le permite al hablante revelar su identidad doble: "Spanglish meets the needs of its speakers in that it allows for the expression of the dual-identity that is the essence of the immigrants' being". El concepto spanglish sigue muy debatido y la reflexión ofrecida por Ana Celia Zentella (2016: 31): "Spanglish is a graphic way of saying "we speak both because we are both"" da cuenta de la complejidad y le lleva a definir el spanglish como un estilo de habla que refleja la naturaleza híbrida: "an in-group and informal style of speaking among Spanish-English bilinguals that honors the rules of both Spanish and English - an act of "doing being bilingual" that reflects our dual worlds" (Zentella 2016: 31).

Muy en general, el cambio de código se define como una alternancia de idiomas en un mismo texto o discurso. Suelen distinguirse tres tipos de alternancias: el 
cambio de código intersentencial, intrasentencial y el tag-switching. El primer tipo, el cambio intersentencial, se refiere a un cambio de lengua entre dos oraciones consecutivas, pues la primera oración aparece en un código, la siguiente en otro. El segundo tipo, el cambio intrasentencial, indica una alternancia dentro de una misma oración. En este caso ciertos constituyentes de una oración aparecen en otro código. El último tipo, el tag-switching o cambio de código emblemático, se identifica como un cambio entre una oración y un tag, o sea un constituyente de alta movilidad que puede insertarse sin afectar la estructura de la oración en la que aparece (cf. Milroy y Muysken 1995; Poplack 1980, etc.). Según Shana Poplack (1980: 589), la alternancia intrasentencial puede considerarse como una forma más compleja o íntima, porque requiere una sinergia, una aplicación conjunta de reglas gramaticales de dos sistemas lingüísticos diferentes: "a codeswitched segment, and those around it, must conform to the underlying syntactic rules of two languages which bridge constituents and link them together grammatically".

Junto a esto, cabe señalar el modelo del Marco de la Lengua Matriz (Matrix Language Frame) desarrollado por Carol Myers-Scotton (2001), según el cual conviene determinar la matrix language o la lengua matriz y la embedded language o la lengua insertada. De acuerdo con este modelo, hay dos códigos activados, pero una lengua asume un papel mayor que la otra. Destaca, pues, el carácter asimétrico del modelo. Ahora bien, la lengua matriz o dominante impone el sistema morfosintáctico y determina si la oración se construye de una manera gramaticalmente correcta, mientras que la lengua insertada asume un rol secundario de inserción en el otro código. Carol Myers-Scotton distingue tres tipos de constituyentes. Por un lado, hay islas de la lengua matriz: son constituyentes formados completamente por morfemas de esta lengua matriz. Por otro lado, hay constituyentes mixtos, tanto de la lengua matriz como de la lengua insertada. Y, para finalizar, hay islas de la lengua insertada que son constituyentes formadas por morfemas de la lengua insertada. Por lo que atañe a la aplicación del sistema gramatical, se procede de la manera siguiente: en el caso de las islas, tanto de la lengua matriz como de la lengua insertada, se sigue el sistema gramatical de la propia lengua; en el caso de los constituyentes mixtos, se suele aplicar el código de la lengua matriz. Así, por ejemplo, en el texto que analizamos con más detención, la lengua matriz es claramente el español y se insertan palabras del inglés.

(1) Edward Hopper meets David Hockney con un twist de Tim Burton para darle sabor. (113) 
En otros textos, por ejemplo en la obra There's No Place Like Home / Camino A Casa Crónica (2008) escrita por Susana Chávez-Silverman, el grado de cambio de código resulta mayor, pero la lengua matriz sigue siendo español. Sirvan de ilustración los fragmentos siguientes:

(2) Over the years, los temas se volverían más personales, todavía candentes, but also tajantemente íntimos: la enfermedad, el cuerpo, los hijos, feeling burnt out, feeling lucky, feeling inspired, feeling. (2)

(3) En vez de mandarme to fly, diciéndome que you didn't have time, o que la poesía no era tu specialty, you sent me a list of names, anotada y con tus recomendaciones. (3)

(4) Quería que me publicaras una entrevista con Paulina. I flooded your Inbox. Te mandé impassioned entreaties, incluyendo detalladas descripciones del current state of Argentine poetry en el milenio. (7)

Dicho esto, procedemos ahora a un análisis detenido de los tipos de cambio de código e intentamos ofrecer una tipificación del texto. El objetivo final del estudio consiste en llegar a una clasificación según el grado de spanglish que el texto presenta. La idea es situar cada texto en una escala continua que tiene en los dos extremos los códigos individuales, español e inglés y en el centro el grado más elevado de cambio de código, o el Spanglish prototípico, si así puede denominarse.

\section{Contextualización del cuento}

Con el objetivo de proporcionar una contextualización del cuento, ofrecemos una breve reflexión sobre quién es el autor. Alberto Fuguet (1964) creció en el estado de California, en los Estados Unidos, antes de trasladarse nuevamente a su país natal: Chile. Antes de dedicarse a la escritura, estudió periodismo en la Universidad de Chile. En 1990 publicó Sobredosis, su primera colección de cuentos. Luego, se publicaron las novelas Mala onda (1991), Tinta roja (1996) y Las películas de mi vida (2004). Su libro más reciente Sudor (2016) recibió comentarios favorables tanto de los críticos como de los lectores. En sus obras literarias desarrolla un auténtico realismo, emplea el lenguaje de la calle (incluso el spanglish) y elabora la cultura popular estadounidense e hispanoamericana. Reflexiones sobre la identidad latinoamericana y los distintos modos en que esta identidad puede construirse caracterizan la obra de Alberto Fuguet, y así, se ha convertido en un hombre de gran influencia no solo en la literatura, sino también en la vida cotidiana (cf. Amar Sánchez 2008; Caro Martín 2007; Medina 2014). 
Y de ahí, Edmundo Paz-Soldán (2002: 43), con quien coeditó Se habla español. Voces latinas en USA (2002), lo considera "uno de los escritores emblemáticos de una generación - nacida en la década del 60 - que ha aparecido en el panorama literario latinoamericano con sorprendente fuerza y energía". Igualmente, "Time/CNN lo eligió como uno de los 50 líderes latinoamericanos del Nuevo Milenio" (Paz-Soldán \& Fuguet 2002: 379).

El cuento Más estrellas que en el cielo que a continuación se analizará en detalle, traza el mundo de las películas en la ciudad de Los Ángeles con los premios Óscar, la alfombra roja y los famosos directores de cine. El cuento mismo describe dos amigos de origen chileno, que toman café en la Cafetería Denny's. Versa sobre gente soñando con ser parte de este glorioso ámbito del cine, de ganar un premio Óscar y de hacer en su discurso público un homenaje a todos los cinematógrafos hispanos. En realidad, el autor está criticando de manera indirecta la sociedad caracterizada por la desigualdad. Como lo indica Ana María Amar Sánchez (2008: 98) el escenario puede verse como ““un encuentro” entre Edward Hopper, Tim Burton y David Hockney: "Estamos en un Denny's con pretensiones estéticas. Edward Hopper meets David Hockney con un twist de Tim Burton para darle sabor" (Fuguet 2000: 113)".

\section{Frecuencia de los códigos español e inglés}

Siguiendo el modelo del Marco de la Lengua Matriz (Matrix Language Frame) desarrollado por Carol Myers-Scotton (2001), pasamos al análisis general de la frecuencia de los códigos español e inglés en el cuento Más estrellas que en el cielo de Alberto Fuguet. En la tabla 1 calculamos cuántas palabras aparecen en cada uno de los códigos.

Tabla 1. Frecuencia de la lengua utilizada

\begin{tabular}{lcc}
\hline Lengua utilizada & \multicolumn{2}{l}{ Frecuencia } \\
\hline Palabras en español & 2300 & $(91,85 \%)$ \\
Palabras en inglés & 204 & $(8,15 \%)$ \\
Palabras en código combinado & 1 & $(<1 \%)$ \\
\hline
\end{tabular}

Las cifras indican un claro predominio del código español, el $92 \%$ de los constituyentes son palabras en español, el $8 \%$ aparece en inglés y hay un ejemplo 
que aparece en un código combinado, se trata de la palabra brode que puede calificarse como una expresión típicamente spanglish, ya que se trata de un término en un inglés hispanizado. Si ahora intentamos posicionar el texto en un continuo, se situará hacia el extremo de español, como puede verse en la figura 1.

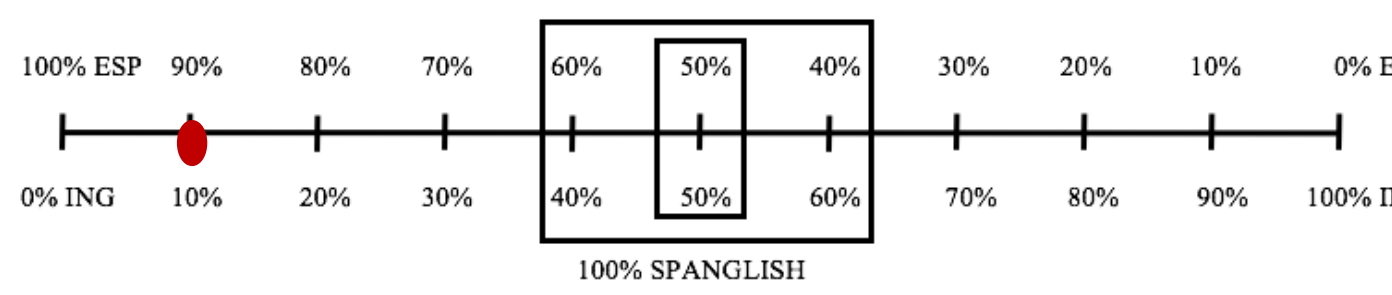

Figura 1. Esquema de las recurrencias

\section{Los distintos tipos de cambio de código en Más estrellas que en el cielo}

Este apartado se centra en las varias formas de cambio de código que aparecen en un caso concreto, el cuento Más estrellas que en el cielo de Alberto Fuguet. A continuación, distinguiremos los tipos siguientes: el cambio de código intersentencial, el cambio intrasentencial y el cambio emblemático.

Por lo que atañe al cambio de código intersentencial, distinguimos entre dos tipos, porque asumen un funcionamiento distinto en el cuento. Por un lado, está el cambio de código intersentencial propiamente dicho. Se trata del cambio de código en el discurso narrativo. Por otro lado, está el tipo conversacional, ya que aparece en las partes dialogadas como enunciados expresados por los protagonistas en el cuento. El primer tipo que aparece como alternancia de frases en el discurso narrativo se ilustra mediante el ejemplo (1):

(1) Denny's, además, es el único antro barato en toda la colina. Talk is cheap, love not. Me queda más que claro. (114)

El segundo tipo de cambio intersentencial que aparece en los diálogos parece tener un uso muy particular, ya que se presenta como alguna manera de identificación. Los protagonistas, en el empleo de la lengua y del Spanglish, se identifican de cierto modo.

(2) - ¿Se puede tomar gin acá?

- It's Oscar night. Todo se puede, todo se debe. (119)

(3) - No - le responde Gregory apenas -. We are with the Chilean delegation.

- The what? 
- La delegación chilena - interrumpo - (121)

Como se ilustra en el ejemplo (3) hay una repetición de la misma idea, pero aparece en una versión traducida.

Otro uso de cambio intersentencial de particular interés se ilustra en los casos siguientes: se trata del empleo de citas en un código distinto.

(4) Acá en el Norte siempre me preguntan: where are you from, man? (114)

(5) Can I get another refill? - insiste el latero de Gregory. (115)

(6) Thanks - le responde molesto, seco, duro-de-matar. (115)

(7) I love her - exclama Farah. (121)

(8) A short movie - agrego. (121)

Pasamos ahora al cambio de código intrasentencial. En estos casos la alternancia se hace en el nivel de los constituyentes de una oración. En el cuento, tenemos oraciones en español con palabras en inglés. Sirvan de ilustración los ejemplos siguientes:

(9) Edward Hopper meets David Hockney con un twist de Tim Burton para darle sabor. (113)

(10) Los dos estamos apoyados en esta barra, sentados sobre unos barstools de cromo. (113-114)

(11) Mucha whipped cream, patatas fritas, racimos de perejil, vasos de hielo con agua. (114)

(12) Es, me informa, de un diseñador muy trendy. (116)

(13) Lázaro nos puso al día rápido: se juntó con productores, hizo mucho network, intercambió e-mails. (118)

(14) Sí, macho, muy boogie nights, muy last dance. (118)

En tercer lugar, comentamos los casos de cambio de código emblemático o tag switching. Se trata de expresiones aisladas de tipo etiquetas como "I mean, you know, sabes, verdad, ..." que aparecen como código alternado. Ahora bien, en el cuento localizamos ejemplos como los siguientes:

(15) Not anymore, carnal. (114)

(16) Las veces que tú quieras, honey. (115)

(17) Cerca, you know, pero no lo suficiente. (116)

A veces se trata de una forma apelativa, carnal en español, honey en inglés. En el tercer ejemplo se inserta el tag you know, lo cual constituye un recurso muy 
frecuentemente empleado en spanglish. Para terminar, hay un ejemplo de particular interés con la palabra brode.

(18) Tú, brode, le temes aléxito. (117)

Esta palabra puede calificarse como una expresión típicamente spanglish, ya que se trata de un término en un inglés hispanizado. Aparece en la lista de términos en spanglish publicada en The Essential Ilan Stavans y significa friend (Stavans 2000b: 32). Esta palabra, como los casos mencionados arriba, aparece también como interjección, como un tag y por su carácter mixto, podría considerarse como el caso más complejo e íntimo de spanglish.

\section{Consideraciones finales}

A modo de conclusión recordamos que el propósito del estudio consiste en establecer indicadores para medir el grado de cambio de código. Se trata de una aproximación basada en un corpus literario de breves cuentos escritos en spanglish. El estudio implica posicionar cada ejemplo, y luego de una manera más general, cada texto, en una escala en la que los dos códigos, español e inglés se sitúan en los puntos extremos y en que la parte central da cuenta de una alternancia máxima, un spanglish al cien por ciento.

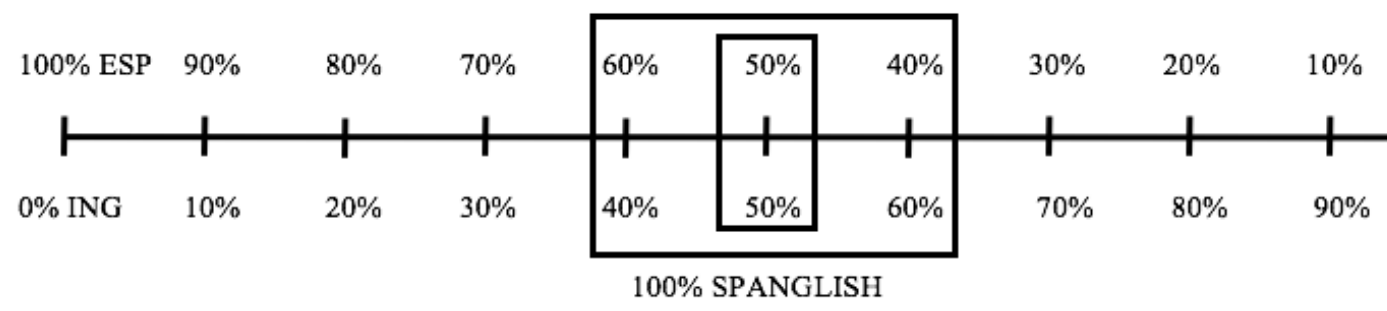

Figura 2. Esquema de análisis

Ahora bien, en este caso concreto sobre el cambio de código en el cuento Más estrellas que en el cielo de Alberto Fuguet, notamos que se sitúa claramente en un extremo. La lengua matriz, el español, es omnipresente y la lengua insertada, el inglés se emplea esencialmente en citas y para indicar ciertos conceptos. Constituye un caso concreto que sirve como ejemplo de cómo intentamos proceder con el análisis de los diferentes cuentos en el corpus compilado para dar cuenta de la variación en el spanglish, de los distintos grados de alternancia. 


\section{Referencias bibliográficas}

Amar Sánchez, A.M. 2008. Del cine al ciberespacio. Narrativa y medios masivos en la tradición literaria latinoamericana. Iberoamericana, VIII, 29, 91-104.

Betti, S. 2009. Spanglish en los Estados Unidos: Apuntes sobre lengua, cultura e identidad. Confluenze, 1, 2, 101-121.

Caro Martín, A. 2007. "América te lo he dado todo y ahora no soy nada". Contracultura y cultura pop norteamericanas en la narrativa de Ray Loriga y Alberto Fuguet. Berlin: LIT Verlag.

Chávez-Silverman, S. 2008. There's No Place Like Home/Camino a Casa Crónica. Journal of Multidisciplinary International Studies, 5(1), 1-9.

Fuguet, A. 2000. Más estrellas que en el cielo. En Paz Soldán, E. \& Fuguet, A. (eds) Se habla español. Voces latinas en USA. Miami: Alfaguara, 111-122.

Medina, H. 2014. Alberto Fuguet y la identidad on the road. El tiempo, el espacio y la identidad en el cuento "Road Story", Cuadernos de literatura, XVIII, 35, 259-282.

Milroy, L. \& Muysken, P. 1995. One Speaker, Two Languages. Cross Disciplinary Perspectives on Code-Switching. Cambridge: University Press.

Myers-Scotton, C. 2001. The matrix language frame model: Developments and responses. En Jacobson, R. (ed.), Codeswitching Worldwide II. Berlin/New York: Mouton de Gruyter, 23-58.

Paz-Soldán, E. \& Fuguet, A. 2002. Se habla español. Voces latinas en USA. Miami: Alfaguara. Poplack, S. 1980. Sometimes I'll start a sentence in Spanish y termino en español: towards a typology of code-switching. Linguistics, 18,7-8, 581-618.

Stavans, I. 2000a. Spanglish: Tickling the Tongue, World Literature Today, 74, 3, 555-559.

Stavans, I. 2000b. The Essential Ilán Stavans. New York: Routledge.

Zentella, A.C. 2016. Spanglish: Language Politics vs el habla del pueblo. En Tamargo, R., Mazak, C. \& Parafita Couto, M.C. Spanish-English Codeswitching in the Carribbean and the U.S., Amsterdam: John Benjamins, 11-35. 